\title{
Genetic Polymorphisms Affecting Tacrolimus Metabolism and the Relationship to Post-Transplant Outcomes in Kidney Transplant Recipients
}

\author{
Fang Cheng ${ }^{1,2}$ \\ Qiang $\mathrm{Li}^{1,2}$ \\ Jinglin Wang ${ }^{1,2}$ \\ Min $\mathrm{Hu}^{1,2}$ \\ Fang Zeng ${ }^{1,2}$ \\ Zhendi Wang ${ }^{3}$ \\ Yu Zhang $\mathbb{D}^{1,2}$
}

'Department of Pharmacy, Union Hospital, Tongji Medical College, Huazhong University of Science and Technology, Wuhan, 430022, People's Republic of China; ${ }^{2}$ Hubei Province Clinical Research Center for Precision Medicine for Critical IIIness, Wuhan, 430022, People's Republic of China; ${ }^{3}$ Department of Urology, Union Hospital, Tongji Medical College, Huazhong University of Science and Technology, Wuhan, 430022, People's Republic of China
Correspondence: Zhendi Wang Department of Urology, Union Hospital, Tongji Medical College, Huazhong University of Science and Technology, Wuhan, 430022, People's Republic of China

Email wangzhendi@gmail.com

\section{Yu Zhang}

Department of Pharmacy, Union Hospital, Tongji Medical College, Huazhong University of Science and Technology, Wuhan, 430022, People's Republic of China

Email zhangwkp@163.com
Background: Tacrolimus is a key drug in kidney transplantation with a narrow therapeutic index. However, whether tacrolimus exposure variability affects clinical outcomes and adverse reactions remains unknown.

Objective: Our study investigated the factors that influence tacrolimus exposure in kidney transplantation recipients and the relationship between tacrolimus concentration and clinical outcomes and adverse reactions.

Settings and Methods: We examined the effect of tacrolimus concentration on clinical outcomes and adverse reactions in 201 kidney transplantation recipients, and identified clinical and pharmacogenetic factors that explain tacrolimus exposure.

Results: The CYP3A5 genotype was clearly associated with dose-adjusted trough blood tacrolimus concentrations $\left(\mathrm{C}_{0} / \mathrm{D}\right)$, whereas no significant difference was observed in patients with the CYP3A4*1B, CYP3A4*22, ABCB1, ABCC2, POR*28 or PXR alleles. Clinical factors such as red blood cell count, hemoglobin, and albumin were the most useful influence factors affecting tacrolimus $\mathrm{C}_{0} / \mathrm{D}$. Besides, Wuzhi capsule increased tacrolimus $\mathrm{C}_{0} / \mathrm{D}$ in kidney transplantation recipients. Furthermore, higher tacrolimus concentrations were associated with higher diarrhea and post-transplant diabetes mellitus (PTDM) risk but not with acute rejection and chronic allograft kidney dysfunction.

Conclusion: Clinical factors, medication, and CYP-enzyme polymorphisms accounted for tacrolimus concentration variability in kidney transplantation recipients. Furthermore, higher tacrolimus concentrations were associated with higher diarrhea and PTDM risk.

Keywords: kidney transplantation, tacrolimus, metabolism, pharmacogenetics, immunosuppression

\section{Introduction}

Kidney transplant recipients require life-long immunosuppression therapy to prevent allograft rejection. ${ }^{1}$ Tacrolimus, an effective and potent calcineurin inhibitor, is a cornerstone of immunosuppression therapy after kidney transplantation. The essential therapeutic effects of tacrolimus, however, are shadowed by its various side effects, narrow therapeutic index and highly variable pharmacokinetic (PK) properties. $^{2}$ Therefore, therapeutic drug monitoring (TDM) is recommended for patients receiving tacrolimus. However, due to individual firstpass effects, the target tacrolimus concentration may lag behind relatively. In addition, the target concentration does not guarantee the therapeutic effect or avoid adverse reactions. 
Several factors can affect the PK properties of tacrolimus, such as diet, drug combinations, genotypes, and clinical factors. In recent years, genetic factors have been considered to play an important role in the interindividual and interethnic variability of tacrolimus. ${ }^{3}$ Tacrolimus is mainly metabolized by cytochrome P450 3A5 (CYP3A5) and CYP3A4 enzymes. ${ }^{4,5}$ Therefore, allelic variation of CYP3A5 and CYP3A4 genes can explain most of the tacrolimus PK variability. Previous studies have well established the influence of CYP3A5 genotype on tacrolimus PK. ${ }^{6,7}$ However, recent studies have shown the limited effectiveness of CYP3A5guided dose adjustment in achieving target concentrations in kidney transplantation recipients, suggesting that additional factors may participate in tacrolimus PK variability. Even though tacrolimus pharmacogenetic/pharmacogenomic studies have only reached a consensus on CYP3A $5 * 3$ as a genetic factor affecting tacrolimus $\mathrm{PK},{ }^{8-10} \mathrm{CYP} 3 \mathrm{~A} 5 * 3$ is believed to account for $40 \%-50 \%$ of variations in tacrolimus dosage requirements, ${ }^{11}$ and the correct dosage remains undetermined for a large proportion of patients. Recent studies have revealed that several other CYP3A4, ABCB1, POR, $\mathrm{ABCC} 2$, and $\mathrm{PXR}$ gene variants that impact tacrolimus metabolism also affect tacrolimus PKs in different transplant populations. ${ }^{2,12,13}$ However, controversies remain about their roles in predicting dose-adjusted trough blood tacrolimus concentrations $\left(\mathrm{C}_{0} / \mathrm{D}\right)$.

The effect of tacrolimus concentration on clinical outcomes and adverse reactions is still a matter of discussion. On the one hand, no correlation was found between tacrolimus trough concentration and biopsy proven acute rejection (BPAR) in the combined renal transplantation cohort. ${ }^{14}$ On the other hand, a study showed that pharmacogenomics and other factors affected the association between tacrolimus trough concentration and clinical outcomes. ${ }^{15} \mathrm{~A}$ meta-analysis showed that CYP3A5 nonexpressers have a significantly increased risk for acute rejection, ${ }^{16}$ but a prospective randomized trial failed to demonstrate that CYP3A5-guided dose adjustment was beneficial for acute rejection, nephrotoxicity, or survival. ${ }^{17}$ In addition, a study has also reported that CYP3A5-guided tacrolimus dosing adjustment did not increase the number of patients reaching therapeutic range and failed to improve clinical outcome. ${ }^{18}$ Therefore, understanding the predictors of tacrolimus PK variability may provide effective and precise dosing strategies for kidney transplantation recipients. Moreover, it is essential to determine whether tacrolimus levels affect clinical outcomes and adverse reactions. Thus, our study investigated the factors influencing tacrolimus PK variability in kidney transplantation recipients and the effect of tacrolimus exposure on clinical outcomes and adverse reactions.

\section{Materials and Methods Patient Selection}

This investigation was a retrospective single-center study, it included 201 kidney transplantation recipients in Union Hospital, Tongji Medical College, Huazhong University of Science and Technology from June 2015 to December 2019. The enrolment criteria were as follows: (a) received tacrolimus, mycophenolate mofetil, and corticosteroids for graft rejection prevention after kidney transplantation, (b) 18-70 years old, (c) complete clinical data. The exclusion criteria were age under 18 years, combined other organs' transplantation, exposure to cyclosporine or intravenous tacrolimus, co-administration of azole antifungal agents, or missing data. The study was approved by the institutional ethics board of Tongji Medical College, Huazhong University of Science and Technology (Wuhan, China) (Number: [2018] S331). This investigation did not interfere with patient diagnosis or the treatment process. Oral informed consent was provided by all patients and approved by the ethics committee, and this study conformed to the ethical standards of the Declaration of Helsinki. In our study, we recruited 201 patients who received kidney transplant from donations after cardiac death (DCD). DCD kidneys were regionally distributed within the organ sharing network system of China, which is similar to the United Network for Organ Sharing. The process of DCD in China has been described previously. ${ }^{19}$ All DCDs were performed by the Organ Procurement Organization of Union Hospital, Tongji Medical College, Huazhong University of Science and Technology, and were conducted in accordance with the Declaration of Istanbul. Information obtained through the electronic medical record system included, demographic data, symptoms, underlying comorbidities, laboratory tests, and clinical medication during peri-operation. Adverse reactions and clinical outcomes were obtained through patient follow-up.

\section{Drug Administration}

All recipients received a calcineurin inhibitor-based triple immunosuppressive regimen (tacrolimus, mycophenolate mofetil, and corticosteroids). Tacrolimus was administered orally under fasting conditions, and the starting dosage was $3.0-5.0 \mathrm{mg}$ twice daily on Day 2 after transplantation. 
Mycophenolate mofetil treatment started at 0.5-1.0 g twice daily on the transplantation day.

\section{Therapeutic Drug Monitoring}

Tacrolimus was administered on the 2nd day after kidney transplantation, and the blood concentration of tacrolimus was detected on the 5th day after the operation.

Drug concentrations were measured twice weekly or more frequently if justified (suspicion of rejection or adverse event). Tacrolimus concentrations were measured in whole blood using the Roche cobas ${ }^{\circledR} \mathrm{e} 411$ electrochemiluminescence analyzer with a range of $0.5-40 \mathrm{ng} / \mathrm{mL}$. The target trough concentration $\left(\mathrm{C}_{0}\right)$ range of tacrolimus in the first month was $8-10 \mathrm{ng} / \mathrm{mL}$ in our hospital. Tacrolimus concentrations were available at 1, 3, 9, and 12-month post-transplant. The effect of clinical and genetic variables on tacrolimus PKs was quantified by examining the changes in tacrolimus $\mathrm{C}_{0} / \mathrm{D}$, a measure used in numerous previous studies.

\section{Genotypes}

Genotyping of polymorphic genes is not routine in the current clinical practice. After therapeutic drug monitoring, we centrifuged the blood samples, removed the supernatant, and precipitated for DNA extraction using the QIAamp DNA Mini Kit (Qiagen, 51306). Genotyping was performed on peripheral blood samples using a Capital Biotechnology Precision Medicine Research Array (CBT-PMRA) Kit (Thermo Fisher Scientific, Waltham, MA, USA) with Applied Biosystems Axiom 2.0 platform. The microarray contained more than 787,400 single nucleotide polymorphisms (SNPs), which included 50,000 novel markers covering East and South Asian populations based on the human genome version 19 (GRCH 37). Microarray experiments were performed following the official standard operating procedures. The whole experiment was divided into five stages and lasted for 4.5 days. In the first phase, DNA amplification lasted 24 h. DNA fragmentation and precipitation were performed on the following day. DNA pellets were then oven-dried and dissolved in a resuspension buffer. DNA concentrations and fragment sizes were tested to assess whether they could be used in subsequent hybridization experiments. Furthermore, DNA hybridization, ligation, staining, washing and array scanning were all performed on the GeneTitan ${ }^{\mathrm{TM}} \mathrm{MC}$ Instrument. Finally, genotyping was performed using the AxiomTM analysis suite v4.0.1. To explain residual PK variability, we selected SNPs that have been reported to significantly affect tacrolimus metabolism, ${ }^{13}$ including (CYP3A5*3 (rs776746),
CYP3A4*1B (rs2740574), CYP3A4*22 (rs35599367), ABCB1 (rs1128503, rs2032582 and rs1045642), ABCC2 (rs717620, rs2273697 and rs3740066), and POR*28 (rs1057868) and PXR (rs6785049).

\section{Clinical Outcomes and Adverse Reactions}

Clinical outcomes include acute rejection (AR) and chronic kidney allograft dysfunction (CKAD) in our study. We defined AR as an acute deterioration of graft function associated with specific pathologic changes in graft biopsies occurring during the first year after transplantation. CKAD was defined according to the guidelines of The Chinese Society of Organ Transplantation of the Chinese Medical Association (CSOT). Our study included the following individual adverse reactions: post-transplant diabetes mellitus (PTDM), secondary infections, liver dysfunction, and diarrhea.

\section{Statistical Analysis}

Categorical variables were described as frequency rates and percentages. When the data were normally distributed, means for continuous variables were described using mean \pm standard deviation. Otherwise, data were presented as the median and interquartile range values. Genotype distribution was evaluated with Hardy-Weinberg equilibrium analysis. Spearman correlation was applied for univariate analysis. Then, the association between genetic and clinical variables of interest and tacrolimus concentration was modeled with multivariate regression analyses. To investigate the relationship between clinical outcomes and adverse reactions and tacrolimus concentration, when the data were normally distributed, means for continuous variables were compared using independent group $t$-tests. Otherwise, the Mann-Whitney test was performed. All statistical analyses were performed using SPSS version 20.0 (IBM Corp., Armonk, NY, USA). P-values $<0.05$ indicated statistically significant differences.

\section{Results}

\section{Patient Characteristics}

Of the 201 patients included in this study, 148 (73.3\%) were males. Demographic data and clinical information of the recipients and donors are shown in Table 1 . The median age of the recipients was 42 years old (interquartile range (IQR) 34-50). Hypertension ( $\mathrm{N}=182$ [90.1\%]) and anemia $(\mathrm{N}=87$ [43.1\%]) were the most common pre-existing conditions. 
Table I Demographic and Clinical Characteristics of Kidney Transplant Recipients and Donors

\begin{tabular}{|c|c|}
\hline Recipients & \\
\hline Gender (male) & $148(73.3)$ \\
\hline Age, years, median (IQR) & $42(34-50)$ \\
\hline $\mathrm{BMI}$, mean $\pm \mathrm{SD}$ & $21.25 \pm 2.38$ \\
\hline \multicolumn{2}{|l|}{ Comorbidity, n (\%) } \\
\hline Hypertension & $182(90.1)$ \\
\hline Anemia & $87(43.1)$ \\
\hline Hepatitis B & II (5.4) \\
\hline Diabetes & II (5.4) \\
\hline Arthrolithiasis & $5(2.5)$ \\
\hline Coronary heart disease, n (\%) & $4(2.0)$ \\
\hline Others & $5(2.5)$ \\
\hline \multicolumn{2}{|l|}{ Laboratory findings, mean $\pm S D$} \\
\hline White blood cell count, $\times 10^{9} / \mathrm{L}$ & $6.13 \pm 1.78$ \\
\hline Red blood cell count, $\times 10^{9} / \mathrm{L}$ & $4.81 \pm 1.97$ \\
\hline Hemoglobin, $g / L$ & $100.89 \pm 22.55$ \\
\hline Hematocrit, \% & $32.64 \pm 8.70$ \\
\hline Platelet count, $\times 10^{9} / \mathrm{L}$ & $179.03 \pm 34.29$ \\
\hline Neutrophil count, $\times 10^{9} / \mathrm{L}$ & $4.19 \pm 1.58$ \\
\hline Total bilirubin, $\mu \mathrm{mol} / \mathrm{L}$ & $8.80 \pm 3.40$ \\
\hline ALT, U/L & $30.28 \pm 8.61$ \\
\hline AST, U/L & $19.81 \pm 4.43$ \\
\hline ALP, U/L & $85.77 \pm 15.40$ \\
\hline TP, g/L & $71.71 \pm 10.74$ \\
\hline ALB, $g / L$ & $43.20 \pm 6.56$ \\
\hline BUN, $\mathrm{mmol} / \mathrm{L}$ & $19.56 \pm 7.12$ \\
\hline Cre, $\mu \mathrm{mol} / \mathrm{L}$ & $945.84 \pm 105.74$ \\
\hline Uric acid, $\mu \mathrm{mol} / \mathrm{L}$ & $373.62 \pm 51.4$ \\
\hline \multicolumn{2}{|l|}{ Donors } \\
\hline Gender (male) & 145 (7I.8) \\
\hline Age & $48(37-58)$ \\
\hline BMI & $24.48 \pm 6.40$ \\
\hline \multicolumn{2}{|l|}{ Immunosuppression regimens } \\
\hline \multicolumn{2}{|l|}{ Induction agent, n (\%) } \\
\hline Basiliximab & 24 (II.9) \\
\hline Antithymocyte globulin & $178(88.1)$ \\
\hline \multicolumn{2}{|l|}{ Antiproliferative agent, $\mathrm{n}(\%)$} \\
\hline Mycophenolate & $194(96.0)$ \\
\hline Azathioprine & $8(4.0)$ \\
\hline Corticosteroid dose, mg, median (IQR) & $32(26-38)$ \\
\hline
\end{tabular}

Abbreviations: IQR, interquartile range; SD, standard deviation; BMI, body mass index; ALT, alanine aminotransferase; AST, aspartate aminotransferase; ALP, alkaline phosphatase; TP, total protein; ALB, albumin; BUN, blood urea nitrogen; Cre, creatinine.

The mean preoperative hemoglobin concentration of the recipients was $100.89 \pm 22.55 \mathrm{~g} / \mathrm{L}$, and that of creatinine was $945.84 \pm 105.74 \mu \mathrm{mol} / \mathrm{L}$. Most patients received induction therapy with antithymocyte globulin $(\mathrm{N}=178$ [88.1\%]), and mycophenolate as an antiproliferative agent $(\mathrm{N}=194$ [96.0\%]). And 79 (39.3\%) patients took Wuzhi capsules (WZC) to increase tacrolimus concentration.

\section{Pharmacogenetic Analysis}

We investigated whether the CYP3A5*3 (rs776746), CYP3A4*22 (rs35599367), CYP3A4*1B (rs2740574), ABCB1 (rs1128503, rs2032582 and rs1045642), ABCC2 (rs717620, rs2273697 and rs3740066), POR28 (rs2868177), and PXR (rs6785049) polymorphisms affected the tacrolimus $\mathrm{C}_{0} / \mathrm{D}$ in the first month after kidney transplantation. The results of the Hardy-Weinberg equilibrium analysis were shown in Table S1, and all genes met the conditions of Hardy-Weinberg equilibrium $(\mathrm{P}>0.05)$.

The individual trend of tacrolimus trough concentration after one-year post-transplantation (Figure 1) showed a wide deviation from the target range of $8-12 \mathrm{ng} / \mathrm{mL}$. We divided the 201 recipients into two groups: CYP3A5 expressers (AA $+\mathrm{AG}, * 1 / * 1+* 1 / * 3)$ and non-expressers (GG, *3/*3). Patients who were CYP3A5 non-expressers had higher tacrolimus trough concentration and $\mathrm{C}_{0} / \mathrm{D}$ than CYP3A5 expressers, which indicated CYP3A5 genotype strongly associated with tacrolimus $\mathrm{C}_{0} / \mathrm{D}$ (Figure 2). However, no significant difference was observed in patients with the ABCB1, ABCC2, POR*28 or PXR alleles (Table 2).

\section{Prognostic Factors of Tacrolimus $C_{0} / D$ in the First Month After Kidney}

\section{Transplantation}

The Pearson correlation analysis showed that recipients' body mass index (BMI), anemia, red blood cell count (RBC), hemoglobin (HGB), albumin (ALB), blood urea nitrogen (BUN), $\mathrm{CYP} 3 \mathrm{~A} 5 * 3 \quad$ (rs776746), $\mathrm{ABCC} 2$ (rs717620), ABCC2 (rs37400660), and WZC might be closely related to tacrolimus $\mathrm{C}_{0} / \mathrm{D}$ (Table S2). Therefore, these 10 factors were included in the multivariate regression analyses, which showed that RBC, HGB, ALB, CYP3A5*3 (rs776746), and WZC were the most useful influence factors affecting tacrolimus $\mathrm{C}_{0} / \mathrm{D}$ (Figure 3 ).

\section{Clinical Outcomes and Adverse Reactions}

Next, we investigated the relationship between adverse reaction occurrence and tacrolimus concentration at different periods (Table 3). Diarrhea occurred in 28 of 201 patients $(13.9 \%)$, mostly during the first month. The 28 


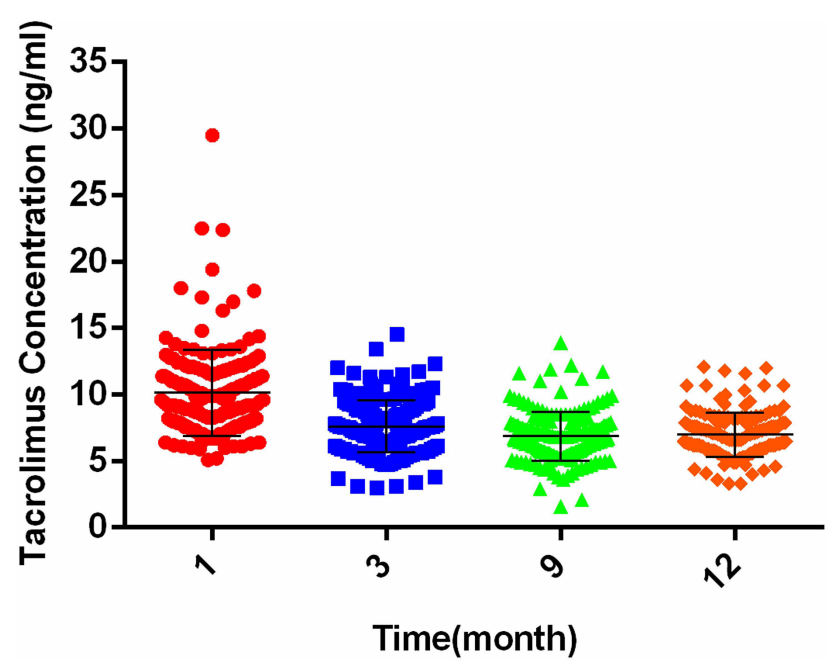

Figure I Tacrolimus concentration distribution in patients at different time interval.

patients with diarrhea had significantly higher tacrolimus concentration than patients without diarrhea. The receiver operating characteristic (ROC) curve of tacrolimus concentration at 1 month after transplantation was statistically significant (area under the curve $(\mathrm{AUC})=0.779, \mathrm{P}<0.001)$. Tacrolimus concentration at 1 month after transplantation $<12.2 \mathrm{ng} / \mathrm{mL}$, which was the best cutoff value for diarrhea. The sensitivity was $67.9 \%$, and the specificity was $90.9 \%$ (Figure 4). At 9-month post-transplantation, tacrolimus concentrations in patients with PTDM were significantly higher than that in patients without PTDM. The ROC curve of tacrolimus concentration at 9-month after transplantation was statistically significant $(\mathrm{AUC}=0.903$, $\mathrm{P}<0.001)$. Tacrolimus concentration at 9-month after transplantation $<9.1 \mathrm{ng} / \mathrm{mL}$, which was the best cutoff value for PTDM. The sensitivity was $72.7 \%$, and the specificity was $94.2 \%$ (Figure 5).

\section{Discussion}

Kidney transplantation recipients usually receive a standard weight-based dose, which is then adjusted based on TDM to maintain tacrolimus blood concentrations within the therapeutic range. However, the correlation between tacrolimus concentration and clinical outcome is still a matter of discussion. This study revealed

A

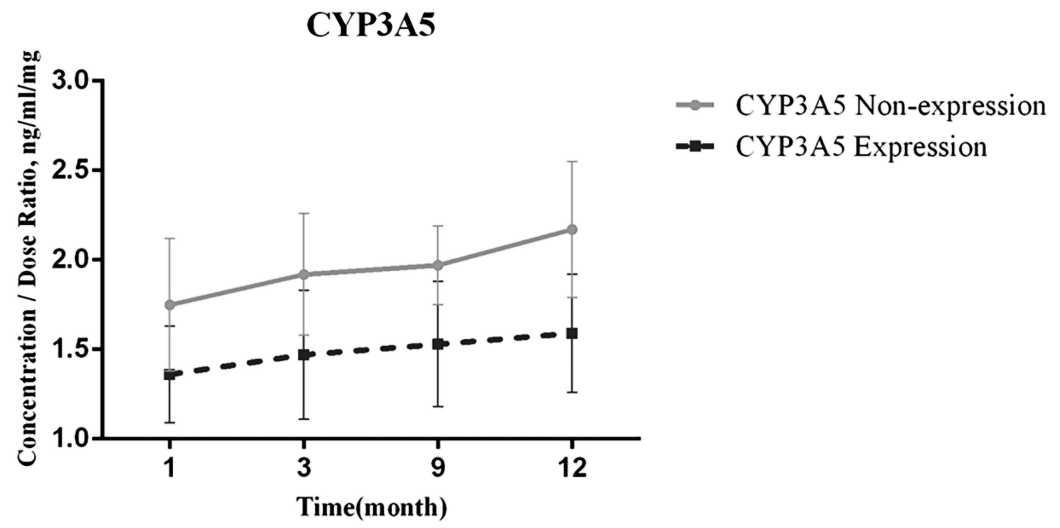

B

CYP3A5

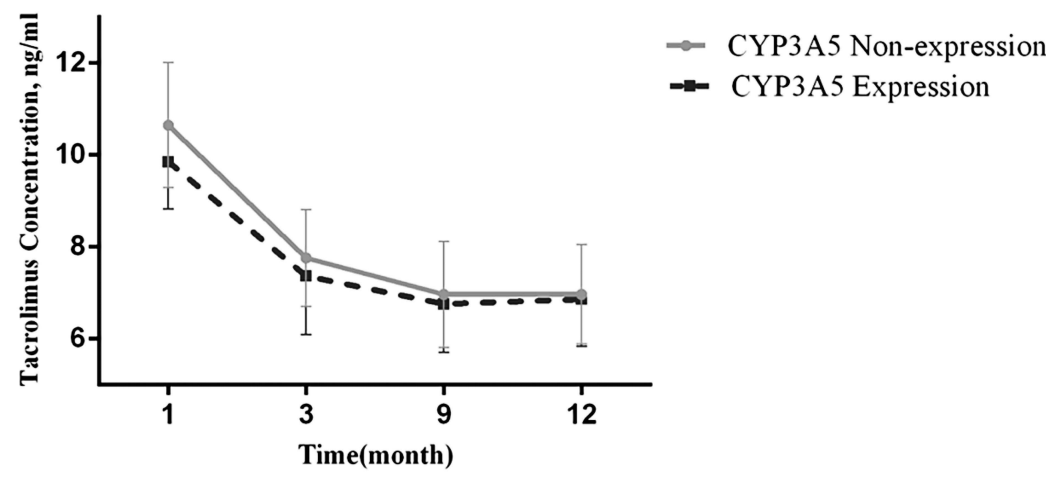

Figure 2 The effect of CYP3A5 genotype on tacrolimus pharmacokinetic (PK) variability. (A) Tacrolimus concentration/dose ratio (Co/D) over time by CYP3A5 genotype. (B) Tacrolimus concentrations over time by CYP3A5 genotype. 
Table 2 Pharmacogenetic Analysis of ABCBI, ABCC2, POR28 and PXR Polymorphism

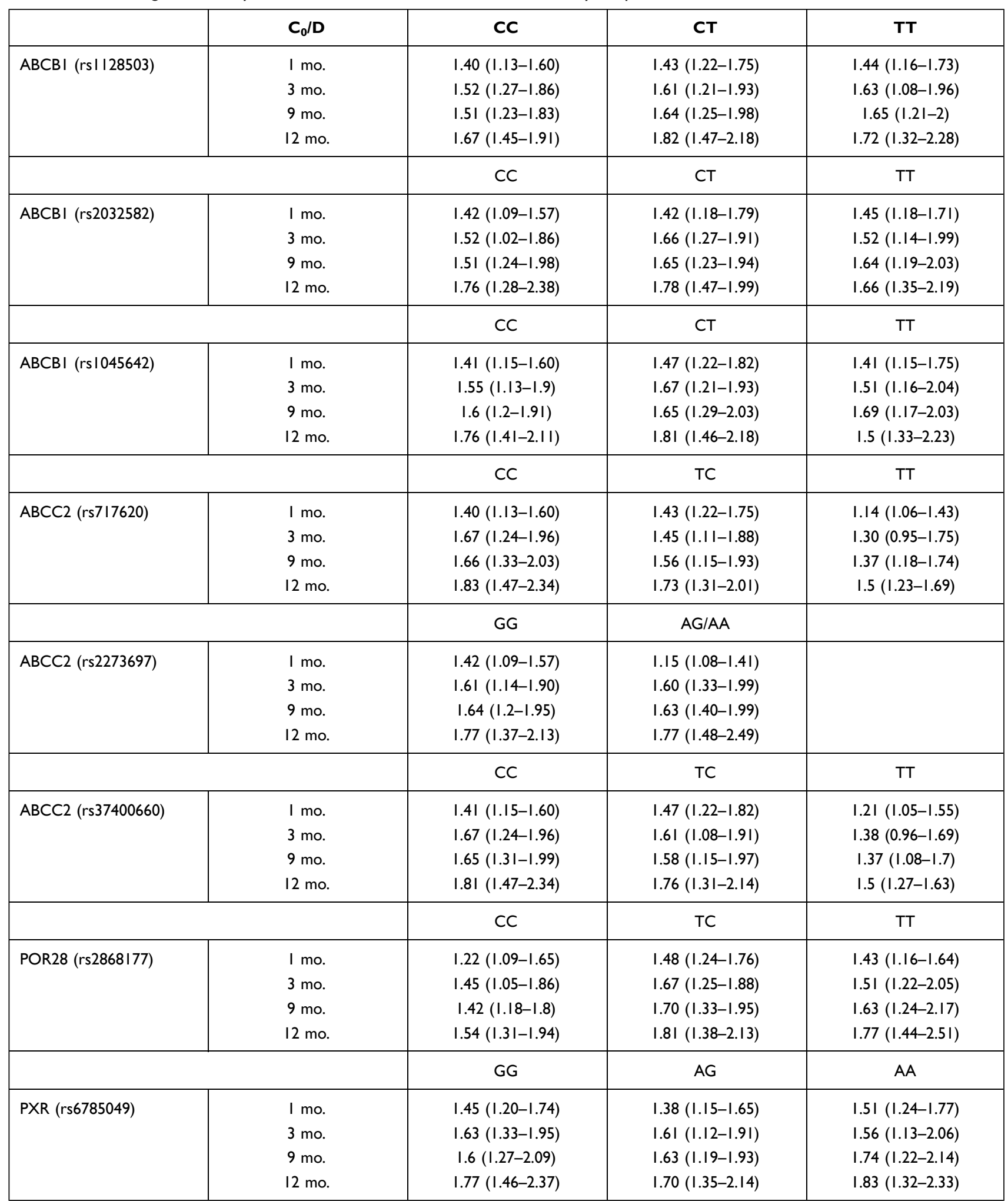

Abbreviations: $A B C B I$, ATP binding cassette subfamily B member I; ABCC2, ATP binding cassette subfamily C member 2; POR, P450 oxidoreductase; PXR, pregnane $X$ receptor. 


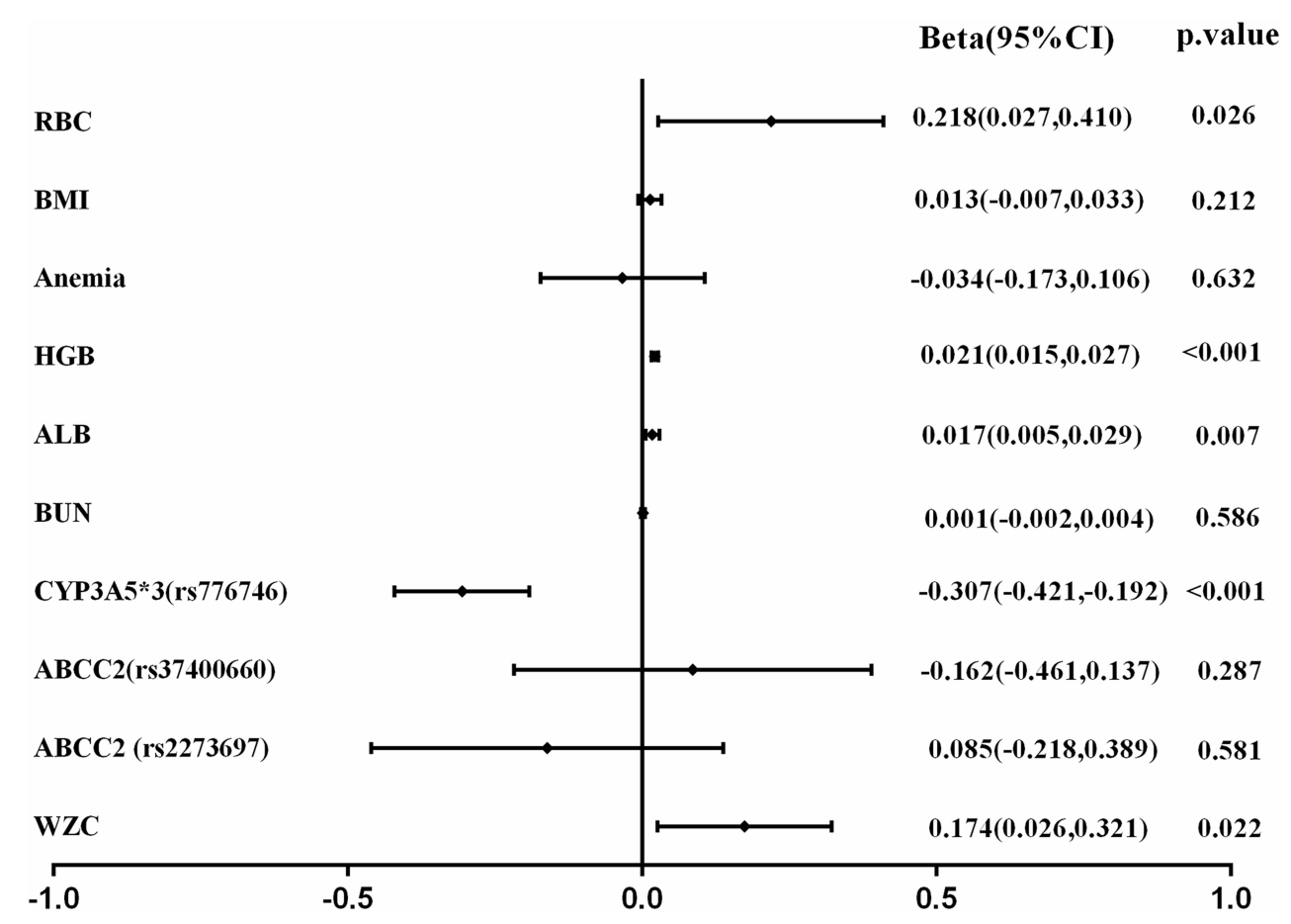

Figure 3 Regression coefficients of multivariable linear regression.

that the CYP3A5 polymorphism was closely associated with tacrolimus $\mathrm{C}_{0} / \mathrm{D}$. We also suggested that clinical variables such as RBC, hemoglobin, albumin, and WZC could explain tacrolimus PK variability. Furthermore, we found that higher tacrolimus concentrations were significantly associated with diarrhea and PTDM risk at different periods. These findings will provide guidance for precision tacrolimus dosing in kidney transplantation recipients.

CYP3A5 is the predominant tacrolimus metabolic pathway, and previous studies have indicated that the CYP3A5 genotype was the most significant source of tacrolimus PK variability. $^{7,20}$ In our study, patients categorized as CYP3A5

Table 3 The Relationship Between Adverse Reactions Occurrence and Tacrolimus Concentration at Different Periods

\begin{tabular}{|c|c|c|c|c|c|}
\hline & & \multicolumn{4}{|c|}{ Tacrolimus Concentration (ng/mL), Median (IQR) } \\
\hline & & I mo. & $3 \mathrm{mo}$. & 9 mo. & 12 mo. \\
\hline \multirow[t]{2}{*}{ AR } & Non-AR & $9.9(8.0-11.6)$ & $7.5(6.3-8.8)$ & $6.8(5.6-8.0)$ & $6.6(5.8-7.9)$ \\
\hline & AR & $8.9(7.3-11.3)$ & $7.3(5.6-8.0)$ & $6.3(5.3-8.3)$ & $7.6(6.4-8.3)$ \\
\hline \multirow[t]{2}{*}{ CKAD } & Non-CKAD & $9.9(7.9-11.5)$ & $7.5(6.3-8.5)$ & $6.8(5.7-7.9)$ & $6.6(5.9-7.9)$ \\
\hline & CKAD & $10.0(8.2-12.0)$ & $7.5(6.2-10.1)$ & $6.8(5.4-8.1)$ & $6.2(5.3-8.4)$ \\
\hline \multirow[t]{2}{*}{ PTDM } & Non-PTDM & $9.9(7.9-11.6)$ & $7.5(6.3-8.5)$ & $6.6(5.6-7.8)^{\mathrm{a} *}$ & $6.6(5.8-8.0)$ \\
\hline & PTDM & $9.4(8.2-11.0)$ & $7.0(5.4-9.7)$ & $10.3(7.5-12.1)$ & $7.0(5.9-8.5)$ \\
\hline \multirow[t]{2}{*}{ Diarrhea } & Non-diarrhea & $9.7(7.7-11.0)^{\mathrm{a} *}$ & $7.5(6.3-8.8)$ & $6.9(5.8-8.1)$ & $6.5(5.7-7.9)$ \\
\hline & Diarrhea & $13.2(9.0-16.6)$ & $7.1(6.1-8.4)$ & $6.2(5.1-7.4)$ & $7.4(6.2-8.3)$ \\
\hline \multirow[t]{2}{*}{ Infections } & Non-infection & 9.8 (7.9-1 I.4) & $7.5(6.3-8.7)$ & $6.8(5.7-7.8)$ & $6.6(5.8-7.8)$ \\
\hline & Infection & $9.9(8.3-12.0)$ & $7.3(6.4-8.8)$ & $6.9(5.1-8.2)$ & $7.6(5.4-8.5)$ \\
\hline \multirow[t]{2}{*}{ Liver dysfunction } & Non-liver dysfunction & $9.9(8.0-11.6)$ & $7.5(6.3-8.7)$ & $6.8(5.6-7.7)$ & $6.6(5.8-7.9)$ \\
\hline & Liver dysfunction & $9.2(7.0-11.4)$ & $6.9(5.6-8.2)$ & $7.3(5.6-7.7)$ & $7.8(5.2-9.1)$ \\
\hline
\end{tabular}

Note: ${ }^{a *} p<0.05$.

Abbreviations: IQR, interquartile range; AR, acute rejection; CKAD, chronic kidney allograft dysfunction; PTDM, post-transplant diabetes mellitus, mo, month. 


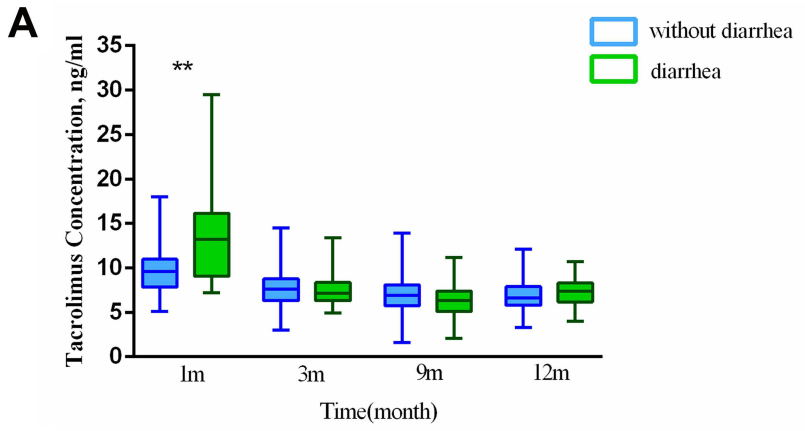

B

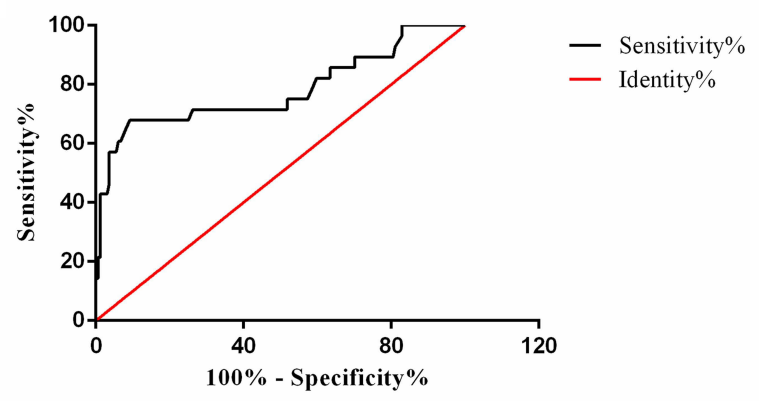

Figure 4 The relationship between diarrhea occurrence and tacrolimus concentration. (A) Tacrolimus concentration over time in the non-diarrhea and diarrhea group. (B) Receiver operating characteristic (ROC) curve. ${ }^{* *} \mathrm{p}<0.01$.

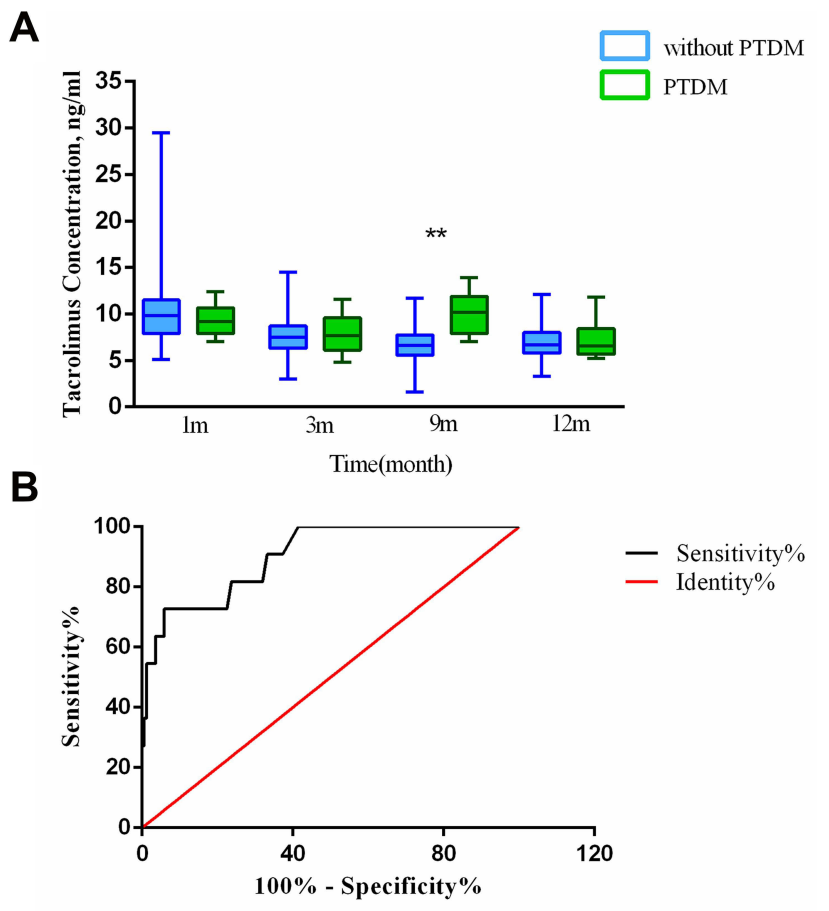

Figure 5 The relationship between post-transplant diabetes mellitus (PTDM) occurrence and tacrolimus concentration. (A) Tacrolimus concentration over time in the nonPTDM and PTDM group. (B) Receiver operating characteristic (ROC) curve. ${ }^{*} \mathrm{p}<0.01$.

non-expresser (poor metabolizers) had higher $\mathrm{C}_{0} / \mathrm{D}$ compared with CYP3A5 expresser (intermediate and extensive metabolizers), which indicated that the CYP3A5 genotype strongly associated with tacrolimus $\mathrm{C}_{0} / \mathrm{D}$. Compared with CYP3A5 polymorphisms, the associations between the tacrolimus PK and polymorphisms in CYP3A4, ABCB1, $\mathrm{ABCC} 2, \mathrm{POR}$, and PXR genes are inconsistent. A previous study suggested that CYP3A4*22 was significantly related to increased tacrolimus concentrations in 1560 EuropeanAmerican kidney transplant recipients. ${ }^{21}$ However, the CYP3A4*22 and CYP3A4*1B SNPs have frequencies of less than $1 \%$ Asian populations. Thus, in our study, no mutation was observed in the CYP3A4*1B allele, and only one patient carried the minor allele of CYP3A4*22. Therefore, the effect of CYP3A $4 * 22$ and CYP3A $4 * 1 \mathrm{~B}$ were not evaluated.

Furthermore, studies involving the relationship between tacrolimus $\mathrm{PKs}$ and $\mathrm{ABCB} 1$ have yielded conflicting results. Some have reported an association between tacrolimus $\mathrm{PK}$ and $\mathrm{ABCB} 1,{ }^{22,23}$ while most found no relationship between $\mathrm{ABCB} 1$ and tacrolimus $\mathrm{C}_{0} / \mathrm{D} .^{24,25} \mathrm{ABCC} 2$ may also be related to tacrolimus metabolism. A study has reported that the $\mathrm{ABCC} 2$ c. $3972 \mathrm{C}>\mathrm{T}$ polymorphism affected tacrolimus $\mathrm{C}_{0} / \mathrm{D}$ in kidney transplant recipients. ${ }^{26}$ However, other studies have reached negative results about the associations between tacrolimus PKs and ABCC2 polymorphisms. ${ }^{23}$ According to our current findings, $\mathrm{ABCB} 1$ and $\mathrm{ABCC} 2$ had no effect on tacrolimus $\mathrm{C}_{0} / \mathrm{D}$ in Chinese kidney transplant recipients.

The POR gene encodes the CYP oxidoreductase, and plays a key role in CYP-mediated drug oxidation. Previous studies have suggested that the POR*28 variant allele decreased tacrolimus exposure in CYP3A5 expressers. ${ }^{18}$ Conversely, some studies have shown that POR*28 did not affect the tacrolimus PKs. ${ }^{27}$ This is consistent with the fact that we did not observe any effect of POR*28 on tacrolimus $\mathrm{C}_{0} / \mathrm{D}$. PXR, encoded by NR1I2, regulates the expression of CYP3A and ABCB1. Studies on the relationship between PXR and tacrolimus PKs yielded conflicting results. ${ }^{28,29}$ However, there were no significant differences observed between POR*28 or PXR alleles and tacrolimus $\mathrm{C}_{0} / \mathrm{D}$ in our study.

Since more than $90 \%$ of tacrolimus in circulation is confined to RBCs, RBC count is expected to affect blood tacrolimus concentration. ${ }^{30}$ Previous studies also reported that tacrolimus was highly bound to plasma proteins, mainly to serum albumin. ${ }^{31}$ Therefore, hemoglobin and albumin influence the free fraction but not the unbound concentration of tacrolimus in blood. Previous studies reported an association between hemoglobin and albumin 
and tacrolimus PKs. ${ }^{32,33}$ A decrease in hemoglobin and albumin levels increases the free fraction of tacrolimus in blood, increasing whole blood clearance, and ultimately resulting in a decrease in tacrolimus blood levels. ${ }^{34}$ These changes are important for the correct interpretation of TDM results. Consistent with prior studies in kidney and other transplant populations, we observed that low RBC, hemoglobin, and albumin levels would result in a low tacrolimus $\mathrm{C}_{0} / \mathrm{D}$.

Tacrolimus is a substrate of P-gp and is mainly metabolized by CYP3A in the liver and small intestine. Therefore, investigating the potential drug interaction effects is necessary before considering combination therapy. Previous study demonstrated that pharmacokinetic interaction occurs between steroids and tacrolimus in renal transplant patients. ${ }^{35}$ Although all transplant patients in our study received the same standard therapeutic regimen (tacrolimus, mycophenolate mofetil, and corticosteroids), there were dose differences in corticosteroids. Therefore, an effect on tacrolimus PKs cannot be ruled out. Azole antifungal agents, such as fluconazole, itraconazole, and posaconazole, could inhibit the activity of CYP3A enzymes and thus reduce tacrolimus clearance, increasing tacrolimus concentrations. ${ }^{36}$ However, kidney transplant patients of our hospital rarely used azoles; therefore, we excluded patients using azole antifungal drugs. In China, a traditional Chinese medicine named Wuzhi capsules (WZC) (registration number in China: Z20025766) is often prescribed to increase tacrolimus concentration in clinical practice. ${ }^{37}$ In vivo and in vitro PK studies demonstrated that WZC inhibited P-gp-mediated efflux and CYP3A-mediated metabolism of tacrolimus, and increased tacrolimus oral bioavailability. ${ }^{38-40}$ Our results also suggested that WZC significantly increased tacrolimus $\mathrm{C}_{0} / \mathrm{D}$. In addition, we did not consider the impact of calcium channel blockers (amlodipine and nifedipine, used for treating hypertension), and this should be clarified in future large-scale studies.

Tacrolimus therapy is associated with risks of nephrotoxicity, neurotoxicity, hypertension, dyslipidemia, diarrhea, and PTDM. ${ }^{41-43}$ The clinical efficacy of any dose of tacrolimus administered for kidney transplantation recipients depends on whether exposure variability negatively impacts outcomes and adverse reactions. However, the correlation between tacrolimus concentration and clinical outcome is still in discussion. Low tacrolimus exposure may increase the risk of acute rejection in the first year after kidney transplantation. ${ }^{44}$ In addition, tacrolimus trough concentrations above $9.3 \mathrm{ng} /$ $\mathrm{mL}$ were significantly associated with higher BPAR risk in the first three months after transplantation. ${ }^{45}$ However, other studies found no significant difference in acute rejection between tacrolimus trough concentrations in the first year or the first two weeks after transplantation, respectively. ${ }^{46,47}$ Our study revealed no association between differential tacrolimus exposure and AR and CKAD after one-year post-transplantation, but the number of patients was small and the follow-up was short. However, we found that tacrolimus concentrations at different periods were significantly associated with diarrhea and PTDM.

Mycophenolate mofetil (MMF) is often considered to be the cause of unexplained diarrhea in kidney transplantation recipients. However, recent studies suggest that systemic exposures to MMF and its metabolites are not associated with diarrhea in liver transplant recipients. ${ }^{48}$ Diarrhea is also a frequent side effect of tacrolimus, but the relationship between tacrolimus and diarrhea has not attracted much attention. In clinical trials, diarrhea was reported in $22-72 \%$ of recipients taking tacrolimus. ${ }^{49}$ Previous studies suggested that the increased diarrhea associated with the combination of tacrolimus and MMF as compared with cyclosporine A and MMF may therefore not simply be caused by increased exposure to MMF. ${ }^{50}$ In addition, diarrhea further increases tacrolimus exposure, fueling a vicious cycle. ${ }^{51}$ Our results showed that diarrhea was correlated with tacrolimus concentration in the first month after transplantation, which was also consistent with the previous study.

A tacrolimus concentration of $12.2 \mathrm{ng} / \mathrm{mL}$ was the best cutoff value for diarrhea. At present, the mechanism of tacrolimus-induced diarrhea is not clear. Tacrolimus can affect intestinal transport and barrier function, thus affecting glucose absorption. ${ }^{52}$ However, diarrhea can damage intestinal epithelial cells, reducing the activity of CYP3A4 and CYP3A5 metabolic enzymes in the local intestine, and decreasing the expression of P-gp or its activity, thereby affecting the metabolism and absorption of tacrolimus, and leading to an increase of tacrolimus concentration. ${ }^{51}$ Confirming whether higher tacrolimus concentrations cause diarrhea or diarrhea increases tacrolimus concentrations requires further studies.

Recent studies have shown that the incidence of PTDM after transplantation is approximately $16 \%-61 \%,{ }^{53}$ depending on medical centers. PTDM is associated with poorer graft function and all-cause mortality as well as an 
increased risk of infections and cardiovascular events. ${ }^{54}$ Higher tacrolimus levels were consistently considered as an important risk factor for PTDM. We also found that high tacrolimus levels in 9 months post-transplantation were related to an increased risk of PTDM. The tacrolimus concentration at 9 months after transplantation of $9.1 \mathrm{ng} /$ $\mathrm{mL}$ was the best cutoff value for PTDM.

\section{Conclusion and Relevance}

Clinical factors, medication, and CYP-enzyme polymorphisms accounted for tacrolimus concentration variability in kidney transplantation recipients. Furthermore, high tacrolimus concentrations were associated with diarrhea and PTDM risk. However, our study has some limitations. First, it is a retrospective analysis study. This is a single-center study with relatively limited sample size. Multicenter and larger long-term studies would better account for the validity of our results. Secondly, we only studied SNPs that may affect tacrolimus metabolism as previously reported. Genomewide studies may identify additional predictive SNPs. Moreover, although all our transplant patients received the same standard therapeutic regimen, food and additional medications may impact tacrolimus PKs and have consequences on outcome variables. For example, all the patients received different doses of a combination of corticosteroids, known to induce cytochrome P450 expression, which may have affected tacrolimus PK may have been affected. In addition, we did not routinely detect the mycophenolate mofetil concentration. Thus, we cannot rule out the effect of mycophenolate mofetil on diarrhea, which requires further research.

\section{Abbreviation}

PK, pharmacokinetic; TDM, therapeutic drug monitoring; CYP, cytochrome P450; AR, acute rejection; BPAR, lower biopsy-proven acute rejection; $\mathrm{CKAD}$, chronic kidney allograft dysfunction; ABCB1, ATP-binding cassette subfamily B member 1; POR, P450 oxidoreductase; ABCC2, ATPbinding cassette subfamily $\mathrm{C}$ member 2; PXR, pregnane $\mathrm{X}$ receptor; NR1I2, nuclear receptor subfamily 1 group I member 2; BPAR, biopsy-proven acute rejection; $\mathrm{C}_{0} / \mathrm{D}$, dose-adjusted trough blood tacrolimus concentrations; DCD, donations after cardiac death; PTDM, post-transplant diabetes mellitus; AUC, Area Under Curve; ROC, receiveroperating characteristic curve; WZC, Wuzhi capsules; RBC, red blood counts; BMI, body mass index; ALT, alanine aminotransferase; AST, aspartate aminotransferase; ALP, alkaline phosphatase; TP, total protein; ALB, albumin;
BUN, blood urea nitrogen; Cre, creatinine; SNP, single nucleotide polymorphism; P-gp, P-glycoprotein; MMF, Mycophenolate mofetil; IQR, interquartile range.

\section{Data Sharing Statement}

The data that support the findings of this study are available from the corresponding author upon reasonable request.

\section{Author Contributions}

All authors made substantial contributions to conception and design, acquisition of data, or analysis and interpretation of data; took part in drafting the article or revising it critically for important intellectual content; agreed to submit to the current journal; gave final approval for the version to be published; and agreed to be accountable for all aspects of the work.

\section{Funding}

This work was supported by the National Key R\&D Program of China (2017YFC0909900).

\section{Disclosure}

The authors have no relevant affiliations, non-financial and financial involvement with any organization or entity with a financial interest in or financial conflict with the subject matter or materials discussed in the manuscript. This includes employment, consultancies, honoraria, stock ownership or options, expert testimony, grants, or patents received or pending, or royalties.

\section{References}

1. Kasiske BL, Zeier MG, Chapman JR, et al. KDIGO clinical practice guideline for the care of kidney transplant recipients: a summary. Kidney Int. 2010;77(4):299-311. doi:10.1038/ki.2009.377

2. Staatz CE, Tett SE. Clinical pharmacokinetics and pharmacodynamics of tacrolimus in solid organ transplantation. Clin Pharmacokinet. 2004;43(10):623-653. doi:10.2165/00003088-200443100-00001

3. Degraeve AL, Moudio S, Haufroid V, et al. Predictors of tacrolimus pharmacokinetic variability: current evidences and future perspectives. Expert Opin Drug Metab Toxicol. 2020;16(9):769-782. doi:10.1080/ 17425255.2020.1803277

4. Tron C, Lemaitre F, Verstuyft C, Petitcollin A, Verdier MC, Bellissant E. Pharmacogenetics of membrane transporters of tacrolimus in solid organ transplantation. Clin Pharmacokinet. 2019;58 (5):593-613. doi:10.1007/s40262-018-0717-7

5. Zhang X, Lin G, Tan L, Li J. Current progress of tacrolimus dosing in solid organ transplant recipients: pharmacogenetic considerations. Biomed Pharmacother. 2018;102:107-114. doi:10.1016/j.biopha.2018.03.054

6. Komine N, Satoh S, Saito M, et al. Influence of CYP3A5 genetic differences in tacrolimus on quantitative interstitial fibrosis and long-term graft function in kidney transplant recipients. Int Immunopharmacol. 2018;58:57-63. doi:10.1016/j.intimp.2018.03.004 
7. Anutrakulchai S, Pongskul C, Kritmetapak K, Limwattananon C, Vannaprasaht $\mathrm{S}$. Therapeutic concentration achievement and allograft survival comparing usage of conventional tacrolimus doses and CYP3A5 genotype-guided doses in renal transplantation patients. $\mathrm{Br}$ J Clin Pharmacol. 2019;85(9):1964-1973. doi:10.1111/bcp.13980

8. Miura M, Satoh S, Kagaya $\mathrm{H}$, et al. Impact of the CYP3A4*1G polymorphism and its combination with CYP3A5 genotypes on tacrolimus pharmacokinetics in renal transplant patients. Pharmacogenomics. 2011;12(7):977-984. doi:10.2217/pgs.11.33

9. Zuo XC, Ng CM, Barrett JS, et al. Effects of CYP3A4 and CYP3A5 polymorphisms on tacrolimus pharmacokinetics in Chinese adult renal transplant recipients: a population pharmacokinetic analysis. Pharmacogenet Genomics. 2013;23(5):251-261. doi:10.1097/ FPC.0b013e32835fcbb6

10. Furuse M, Hosomi S, Nishida $\mathrm{Y}$, et al. The impact of cytochrome P450 3A genetic polymorphisms on tacrolimus pharmacokinetics in ulcerative colitis patients. PLoS One. 2021;16(4):e250597. doi:10.1371/journal.pone.0250597

11. Birdwell KA, Decker B, Barbarino JM, et al. Clinical Pharmacogenetics Implementation Consortium (CPIC) guidelines for CYP3A5 genotype and tacrolimus dosing. Clin Pharmacol Ther. 2015;98(1):19-24. doi:10.1002/cpt.113

12. Elens L, Capron A, van Schaik RH, et al. Impact of CYP3A4*22 allele on tacrolimus pharmacokinetics in early period after renal transplantation: toward updated genotype-based dosage guidelines. Ther Drug Monit. 2013;35(5):608-616. doi:10.1097/FTD.0b013e318296045b

13. Lunde I, Bremer S, Midtvedt K, et al. The influence of CYP3A, PPARA, and POR genetic variants on the pharmacokinetics of tacrolimus and cyclosporine in renal transplant recipients. Eur J Clin Pharmacol. 2014;70(6):685-693. doi:10.1007/s00228-014-1656-3

14. Bouamar R, Shuker N, Hesselink DA, et al. Tacrolimus predose concentrations do not predict the risk of acute rejection after renal transplantation: a pooled analysis from three randomized-controlled clinical trials $(\dagger) . A m$ J Transplant. 2013;13(5):1253-1261. doi:10.1111/ajt.12191

15. Taber DJ, Gebregziabher MG, Srinivas TR, Chavin KD, Baliga PK, Egede LE. African-American race modifies the influence of tacrolimus concentrations on acute rejection and toxicity in kidney transplant recipients. Pharmacotherapy. 2015;35(6):569-577. doi:10.1002/phar.1591

16. Rojas L, Neumann I, Herrero MJ, et al. Effect of CYP3A $5 * 3$ on kidney transplant recipients treated with tacrolimus: a systematic review and meta-analysis of observational studies. Pharmacogenomics J. 2015;15 (1):38-48. doi:10.1038/tpj.2014.38

17. Thervet E, Loriot MA, Barbier S, et al. Optimization of initial tacrolimus dose using pharmacogenetic testing. Clin Pharmacol Ther. 2010;87(6):721-726. doi:10.1038/clpt.2010.17

18. Shuker N, Bouamar R, van Schaik RH, et al. A randomized controlled trial comparing the efficacy of Cyp3a5 genotype-based with body-weight-based tacrolimus dosing after living donor kidney transplantation. Am J Transplant. 2016;16(7):2085-2096. doi:10.1111/ajt.13691

19. Summers DM, Watson CJ, Pettigrew GJ, et al. Kidney donation after circulatory death (DCD): state of the art. Kidney Int. 2015;88 (2):241-249. doi:10.1038/ki.2015.88

20. van Gelder T, Meziyerh S, Swen JJ, de Vries APJ, Moes D. The clinical impact of the C(0)/D ratio and the CYP3A5 genotype on outcome in tacrolimus treated kidney transplant recipients. Front Pharmacol. 2020;11:1142. doi:10.3389/fphar.2020.01142

21. Hannachi I, Ben Fredj N, Chadli Z, et al. Effect of CYP3A4*22 and CYP3A4*1B but not CYP3A5*3 polymorphisms on tacrolimus pharmacokinetic model in Tunisian kidney transplant. Toxicol Appl Pharmacol. 2020;396:115000. doi:10.1016/j.taap.2020.115000

22. Cusinato DA, Lacchini R, Romao EA, Moysés-Neto M, Coelho EB. Relationship of CYP3A5 genotype and ABCB1 diplotype to tacrolimus disposition in Brazilian kidney transplant patients. $\mathrm{Br} J$ Clin Pharmacol. 2014;78(2):364-372. doi:10.1111/bcp.12345
23. Pulk RA, Schladt DS, Oetting WS, et al. Multigene predictors of tacrolimus exposure in kidney transplant recipients. Pharmacogenomics. 2015;16(8):841-854. doi:10.2217/pgs.15.42

24. Li JL, Liu S, Fu Q, et al. Interactive effects of CYP3A4, CYP3A5, MDR1 and NR1I2 polymorphisms on tacrolimus trough concentrations in early postrenal transplant recipients. Pharmacogenomics. 2015;16(12):1355-1365. doi:10.2217/pgs.15.78

25. Oetting WS, Wu B, Schladt DP, et al. Attempted validation of 44 reported SNPs associated with tacrolimus troughs in a cohort of kidney allograft recipients. Pharmacogenomics. 2018;19 (3):175-184. doi:10.2217/pgs-2017-0187

26. Genvigir FDV, Nishikawa AM, Felipe CR, et al. Influence of $\mathrm{ABCC} 2, \mathrm{CYP} 2 \mathrm{C} 8$, and CYP2J2 polymorphisms on tacrolimus and mycophenolate sodium-based treatment in Brazilian kidney transplant recipients. Pharmacotherapy. 2017;37(5):535-545. doi:10.1002/phar. 1928

27. Jannot AS, Vuillemin X, Etienne I, et al. A lack of significant effect of POR*28 allelic variant on tacrolimus exposure in kidney transplant recipients. Ther Drug Monit. 2016;38(2):223-229. doi:10.1097/ FTD.0000000000000267

28. Stifft F, van Kuijk SMJ, Bekers O, Christiaans MHL. Increase in tacrolimus exposure after steroid tapering is influenced by CYP3A5 and pregnane $\mathrm{X}$ receptor genetic polymorphisms in renal transplant recipients. Nephrol Dial Transplant. 2018;33(9):1668-1675. doi:10.1093/ndt/gfy096

29. Knops N, Levtchenko E, van den Heuvel B, Kuypers D. From gut to kidney: transporting and metabolizing calcineurin-inhibitors in solid organ transplantation. Int $J$ Pharm. 2013;452(1-2):14-35. doi:10.1016/j.ijpharm.2013.05.033

30. Spallanzani V, Bindi L, Bianco I, et al. Red blood cell exchange as an approach for treating a case of severe tacrolimus overexposure. Transfus Apher Sci. 2017;56(2):238-240. doi:10.1016/j.transci.2017.01.004

31. Brown NW, Gonde CE, Adams JE, Tredger JM. Low hematocrit and serum albumin concentrations underlie the overestimation of tacrolimus concentrations by microparticle enzyme immunoassay versus liquid chromatography-tandem mass spectrometry. Clin Chem. 2005;51(3):586-592.

32. Stratta P, Quaglia M, Cena T, et al. The interactions of age, sex, body mass index, genetics, and steroid weight-based doses on tacrolimus dosing requirement after adult kidney transplantation. Eur J Clin Pharmacol. 2012;68(5):671-680. doi:10.1007/s00228-011-1150-0

33. Zahir H, McCaughan G, Gleeson M, Nand RA, McLachlan AJ. Factors affecting variability in distribution of tacrolimus in liver transplant recipients. Br J Clin Pharmacol. 2004;57(3):298-309. doi:10.1046/j.1365-2125.2003.02008.x

34. Chow FS, Piekoszewski W, Jusko WJ. Effect of hematocrit and albumin concentration on hepatic clearance of tacrolimus (FK506) during rabbit liver perfusion. Drug Metab Dispos. 1997;25 (5):610-616.

35. Anglicheau D, Flamant M, Schlageter MH, et al. Pharmacokinetic interaction between corticosteroids and tacrolimus after renal transplantation. Nephrol Dial Transplant. 2003;18(11):2409-2414. doi:10.1093/ndt/gfg381

36. Vanhove T, Bouwsma H, Hilbrands L, et al. Determinants of the magnitude of interaction between tacrolimus and voriconazole/posaconazole in solid organ recipients. Am J Transplant. 2017;17 (9):2372-2380. doi:10.1111/ajt.14232

37. Chen X, Wang DD, Xu H, Li ZP. Initial dosage optimization of tacrolimus in Chinese pediatric patients undergoing kidney transplantation based on population pharmacokinetics and pharmacogenetics. Expert Rev Clin Pharmacol. 2020;13(5):553-561. doi:10.1080/ 17512433.2020.1767592

38. Chen X, Wang D, Xu H, Li Z. Initial dose optimization of tacrolimus for children with systemic lupus erythematosus based on the CYP3A5 polymorphism and coadministration with Wuzhi capsule. J Clin Pharm Ther. 2020;45(2):309-317. 
39. Yan L, Yang ZQ, Shi YY, et al. Effects of wuzhi capsules on blood concentration of tacrolimus in renal transplant recipients. Ann Transplant. 2019;24:594-604. doi:10.12659/AOT.918980

40. Cheng F, Li Q, Wang J, Zeng F, Zhang Y. Effects and safety evaluation of wuzhi capsules combined with tacrolimus for the treatment of kidney transplantation recipients. J Clin Pharm Ther. 2021;46 (6):1636-1649. doi:10.1111/jcpt.13493

41. Vandenbussche C, Van der Hauwaert C, Dewaeles E, et al. Tacrolimus-induced nephrotoxicity in mice is associated with microRNA deregulation. Arch Toxicol. 2018;92(4):1539-1550. doi:10.1007/s00204-018-2158-3

42. Mittal N, Thompson JF, Kato T, Tzakis AG. Tacrolimus and diarrhea: pathogenesis of altered metabolism. Pediatr Transplant. 2001;5 (2):75-79. doi:10.1034/j.1399-3046.2001.005002075.x

43. Hoorn EJ, Walsh SB, McCormick JA, et al. The calcineurin inhibitor tacrolimus activates the renal sodium chloride cotransporter to cause hypertension. Nat Med. 2011;17(10):1304-1309. doi:10.1038/ nm. 2497

44. Davis S, Gralla J, Klem P, et al. Lower tacrolimus exposure and time in therapeutic range increase the risk of de novo donor-specific antibodies in the first year of kidney transplantation. Am J Transplant. 2018;18(4):907-915. doi:10.1111/ajt.14504

45. Borobia AM, Romero I, Jimenez C, et al. Trough tacrolimus concentrations in the first week after kidney transplantation are related to acute rejection. Ther Drug Monit. 2009;31(4):436-442. doi:10.1097/ FTD.0b013e3181a8f02a

46. Brunet M, van Gelder T, Åsberg A, et al. Therapeutic drug monitoring of tacrolimus-personalized therapy: second consensus report. Ther Drug Monit. 2019;41(3):261-307. doi:10.1097/FTD.0000000000000640

47. Hu R, Barratt DT, Coller JK, Sallustio BC, Somogyi AA. Effect of tacrolimus dispositional genetics on acute rejection in the first 2 weeks and estimated glomerular filtration rate in the first 3 months following kidney transplantation. Pharmacogenet Genomics. 2019;29 (1):9-17. doi:10.1097/FPC.0000000000000360
48. Xia ZW, Jun CY, Hao C, Bing C, Min SM, Jie XJ. The occurrence of diarrhea not related to the pharmacokinetics of MPA and its metabolites in liver transplant patients. Eur J Clin Pharmacol. 2010;66 (7):671-679. doi:10.1007/s00228-010-0833-2

49. Campagne O, Mager DE, Brazeau D, Venuto RC, Tornatore KM. The impact of tacrolimus exposure on extrarenal adverse effects in adult renal transplant recipients. $\mathrm{Br} J$ Clin Pharmacol. 2019;85 (3):516-529. doi:10.1111/bcp.13811

50. Heller T, van Gelder T, Budde K, et al. Plasma concentrations of mycophenolic acid acyl glucuronide are not associated with diarrhea in renal transplant recipients. Am J Transplant. 2007;7(7):1822-1831. doi:10.1111/j.1600-6143.2007.01859.x

51. Lemahieu W, Maes B, Verbeke K, Rutgeerts P, Geboes K, Vanrenterghem Y. Cytochrome P450 3A4 and P-glycoprotein activity and assimilation of tacrolimus in transplant patients with persistent diarrhea. Am J Transplant. 2005;5(6):1383-1391. doi:10.1111/j.16006143.2005.00844.x

52. van Boekel GA, Aarnoutse RE, van der Heijden JJ, Hoogtanders KE, Hilbrands LB. Effect of mild diarrhea on tacrolimus exposure. Transplantation. 2012;94(7):763-767. doi:10.1097/ TP.0b013e3182629e13

53. Hanouneh IA, Feldstein AE, McCullough AJ, et al. The significance of metabolic syndrome in the setting of recurrent hepatitis $\mathrm{C}$ after liver transplantation. Liver Transpl. 2008;14(9):1287-1293. doi:10.1002/lt.21524

54. Watt KD, Pedersen RA, Kremers WK, Heimbach JK, Charlton MR. Evolution of causes and risk factors for mortality post-liver transplant: results of the NIDDK long-term follow-up study. Am J Transplant. 2010;10(6):1420-1427. doi:10.1111/j.1600-6143.2010.03126.x
Pharmacogenomics and Personalized Medicine

\section{Publish your work in this journal}

Pharmacogenomics and Personalized Medicine is an international, peer-reviewed, open access journal characterizing the influence of genotype on pharmacology leading to the development of personalized treatment programs and individualized drug selection for improved safety, efficacy and sustainability. This journal is indexed

\section{Dovepress}

on the American Chemical Society's Chemical Abstracts Service (CAS). The manuscript management system is completely online and includes a very quick and fair peer-review system, which is all easy to use. Visit http://www.dovepress.com/testimonials.php to read real quotes from published authors. 